\title{
Temporary shortage of DPTP-Hib
}

A temporary shortage of DPTP-Hib vaccine has resulted in deferred booster shots and reallocated supplies.

During routine pre-market testing in July, Health Canada uncovered a problem with a batch of Pentacel, a vaccine produced by Aventis Pasteur that protects against diphtheria, per$\checkmark$ tussis, tetanus, polio (DPTP) $\neq$ and Haemophilus influenzae type b (Hib). Children between 2 months and 18 months receive 4 Pentacel shots, followed by a booster (Quadracel) between $\because \vec{H}$ ages 4 and 6 .

$\stackrel{\circ}{\circ}$ Health Canada discovered 1 lot of inactivated polio vaccine that was not of the potency required, said Dr. Arlene King, director of Immunization and Respiratory Infection. The vaccine was not distributed. Aventis expects to have a new supply ready by early November.

Meanwhile, Health Canada is working with jurisdictions to manage the shortage by reallocating supply, deferring booster shots, and, if necessary, purchasing other approved products not currently in supply in Canada.

As of Sept. 15, some central supplies of Pentacel remained at Aventis, said King. Manitoba,
Nova Scotia, New Brunswick, Newfoundland and Labrador, Alberta, and some Ontario communities planned to delay booster shots to ensure enough supply for infant inoculations.

When the existing stock of Pentacel vaccine is used, Quadracel plus Hib can be used in combination.

"Our primary national strategy has been that nowhere in the country do we want to jeopardize infant immunization, because the primary series in the first 6 months is critical," King told CMA7. - Pauline Comeau, Ottawa

\section{Childbirth}

\section{Canada achieves lowest perinatal mortality ever}

The increasing medicalization of childbirth in Canada isn't surprising and does have its benefits, says the Society of ObI stetricians and Gynaecologists 索 of Canada (SOGC).

President-elect Dr. Michael Helewa says rising rates of cesarean sections, medical inductions and epidurals identified in

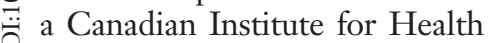
Information (CIHI) report, Giv-

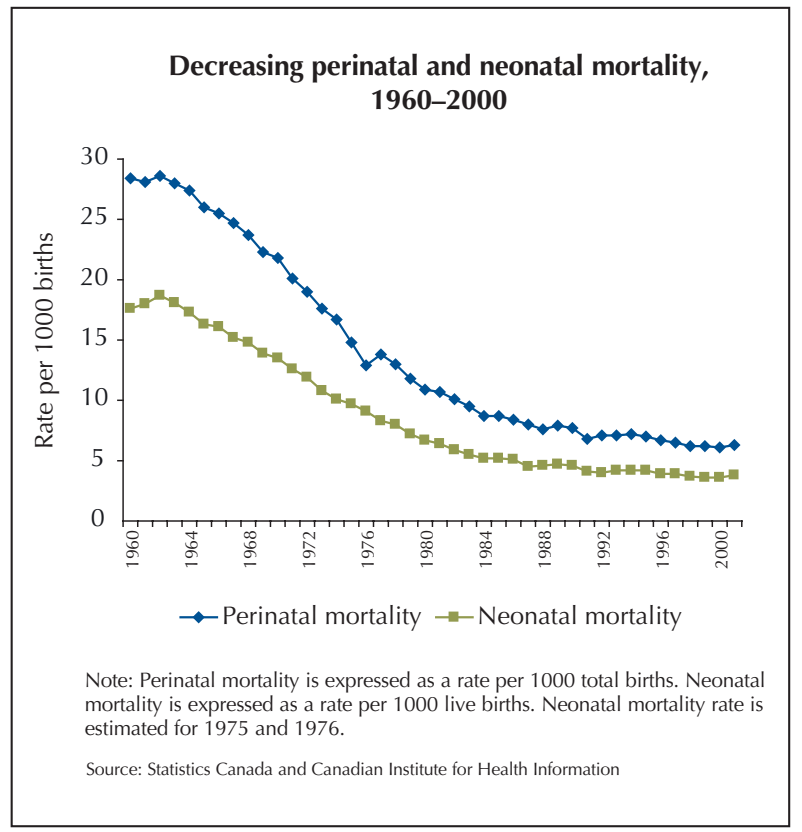

ing Birth in Canada, "has resulted in the lowest perinatal mortality we have ever reached."

According to the CIHI report (www.cihi.ca), $16.5 \%$ of babies born to first-time mothers were delivered by cesarean section in 2002 - up from $13.8 \%$ in 1998 . Epidurals were used in nearly half ( $45.4 \%$ ) of all births in 2001/02, and medically induced births increased substantially to $19.7 \%$ in $2000 / 01$ from $12.9 \%$ a decade earlier.

Helewa says there are complex factors behind these trends, including changes in patient demographics and patient expectations, emerging evidence-based practice, the shift in maternity services from rural to urban areas and the training of physicians, nurses, midwives and health care workers.

The increasing number of women having their first child after they are 35 years of age is having a significant impact on cesarean rates. "With age, for example, comes an increase in the rate of chromosomal anomalies, diabetes, more prolonged and difficult labour and bigger babies," says Helewa, head of clinical obstetrics at St.
Boniface General Hospital in Winnipeg.

Another contributing factor is the increase in maternal obesity, which increases the risk of hypertension and makes it difficult for a baby to pass easily through the birth canal. Other factors include new reproductive technologies that increase chances of multiple births and emerging evidence-based medicine, such as a multicentre trial showing that cesarean section reduced perinatal mortality in breech births. Women are also requesting epidurals more often to avoid pain in labour.

The constant fear of medicolegal action also affects the way physicians and new residents practise, Helewa says, as does the availability of resources. For example, the closure of rural services shifts patients to urban tertiary care centres where high-tech equipment is easily accessible.

CIHI president Glenda Yeates said the institute's report provides the first data of its kind on obstetrical care in Canada and "puts out very hard, reliable data for discussions." - Suzanne Morrison, Oakville, Ont. 